\title{
MONITORING REGENERATIVE HEAT EXCHANGER IN STEAM POWER PLANT BY MAKING USE OF THE RECURRENT NEURAL NETWORK
}

\author{
Tacjana Niksa-Rynkiewicz ${ }^{1, *}$, Natalia Szewczuk-Krypa ${ }^{1}$, Anna Witkowska ${ }^{1}$, \\ Krzysztof Cpałka ${ }^{2}$, Marcin Zalasiński ${ }^{2}$, Andrzej Cader ${ }^{3,4}$ \\ ${ }^{1}$ Gdansk University of Technology, Faculty of Ocean Engineering and Ship Technology, \\ 1112 Gabriela Narutowicza Street, 80-233 Gdansk, Poland \\ ${ }^{2}$ Czestochowa University of Technology, Department of Intelligent Computer Systems \\ Al. Armii Krajowej 36, 42-200 Czestochowa, Poland \\ ${ }^{3}$ University of Social Sciences, \\ Information Technology Institute, Lodz, Poland \\ ${ }^{4}$ Clark University, \\ Worcester, MA 01610, USA \\ *E-mail: marcin.zalasinski@pcz.pl
}

Submitted: 7th July 2020; Accepted: 22th December 2020

\begin{abstract}
Artificial Intelligence algorithms are being increasingly used in industrial applications. Their important function is to support operation of diagnostic systems. This paper presents a new approach to the monitoring of a regenerative heat exchanger in a steam power plant, which is based on a specific use of the Recurrent Neural Network (RNN). The proposed approach was tested using real data. This approach can be easily adapted to similar monitoring applications of other industrial dynamic objects.
\end{abstract}

Keywords: recurrent neural network, intelligent industrial monitoring, Almeida-Pineda recurrent back-propagation, regenerative heat exchanger, steam power plant.

\section{Introduction}

Non-invasive monitoring methods (NDMs) are important from both the theoretical and practical points of view. Therefore, this area is developing in a very dynamic way, and more and more effective algorithms and their applications are being searched for and researched. In NDMs, Artificial Intelligence algorithms are being used increasingly frequently. These methods are more and more often used in industrial applications. They are particularly important for solving those problems where an accurate or fast enough model is not available. Artificial Intelligence algorithms can be used to support an expert in analyzing dynamically changing operating conditions of a given monitored system. Such support might consist in a prompt indication that the system being monitored has reached an unfavorable, usually a dangerous level of its operating condition, which consequently might result in a damage, much quicker wear and tear of the system's components, or some other disadvantageous situations. An immediate detection of unfavorable conditions is par- 
ticularly important in the application range considered and discussed in this work.

This paper focuses on monitoring values of certain selected parameters in a steam power plant system, i.e. the regenerative exchanger and its dependent devices in particular. A faultless operation of the exchanger is important because it significantly affects operational safety of the plant and the power of circulation. It is also a key factor in the efficiency of the system as the efficiency and power of the system are the crucial parameters in every steam power plant. These parameters depend, in particular, on the flow conditions of the condensate through the successive groups of the turbine stages. At the same time, the condensate flow is significantly dependent on the operation of the regeneration exchangers. Therefore, in order to ensure effective and safe operation of the power plant, it is necessary to maintain proper operation of the regenerative heat exchangers and to apply appropriate monitoring systems with the option of detecting undesirable operating conditions.

The proposed approach can be easily adapted to other applications for monitoring the operating conditions of a dynamic system.

\subsection{Related works}

The calculation models used to describe the relationship between the parameters of individual devices of a steam power plant are most often based on the mass and energy balance $[3,4,32]$. However, they are considered to fulfill their function only at the design stage, and are thought to not be suitable for use during the block operation $[15,18,19]$. The main reason mentioned in the literature is the lack of adequate reference data describing the correct and efficient operation of the steam power plant, to which it would be possible to compare the actual parameters of the block operation obtained under variable conditions [9]. The authors of the paper [13] also claim that classical computational models are not suitable for diagnostic applications with high dynamics changes. The reason for this is the inability to generate reference data quickly. Therefore, to overcome this problem, the solutions based on artificial intelligence are commonly applicable. Such solutions include, for example, Artificial Neural Networks (ANN [2, 8, 27, 33]). The paper [30] presents the usefulness of ANNs to de- termine Future Exit Gas Temperature (FEGT) in a $500 \mathrm{MW}$ thermal power plant. The ANN presented in the paper gives a good approximation of the considered system and is a tool that well modulates the non-linear dependence of the boiler outlet gas temperature. The authors claim that the solution which they propose may significantly improve the performance of complex systems of steam power plants. In the study [6], an ANN and a Radial Basis Neural Network (RBNN, [27]) were also used to monitor system parameters in real time. The authors analysed a boiler operating in a thermal power plant of $200 \mathrm{MW}$. They examined the impact of the system parameters on the boiler efficiency and heat rate. They noted that due to a large amount of measurement data generated online, it is not possible to study this impact using classical mathematical models. They underlined the need to create dedicated expert systems for continuous monitoring. Such systems would support operators' decisions to ensure a safe and efficient operation of the system. In the article [14] an ANN was used to detect damage to the steam boiler. The authors focused on the type of damage that would lead to shutting down the boiler and stopping the whole installation. The authors have shown that the network that they used is able to correctly recognize the first symptoms of a faulty operation of the steam boiler, e.g. too low a temperature of the so-called superheated steam. Additionally, they noted that despite a high complexity of the system and many parameters having a mutual influence on each other, the presented ANN worked with a satisfactory speed and accuracy. In [7] the authors used the measurement data obtained from a real system with a rated power of 210 MW. The ANN applied was expected to determine the steam temperature behind the so-called superheater temperature based on the unit load value, amount of fuel feed and feed water flow. In [32] the authors have noted that the ANN can successfully replace traditional steam system models (using mass and energy balances). This article describes the neural model which was supposed to predict the power output of a coal-fired power plant. It was assumed that the model of a power plant was built from two interconnected subsystems: a boiler part and a turbine part. The operation of each subsystems was simulated with a different ANN. The first ANN was supposed to map the behavior of the steam boiler, while the second ANN was to model 
the operation of the steam turbine. The output signals from the first network were given as the inputs for network two. The output parameter of the system of both networks was the predicted power. In the studies carried out, the data from the measurements of real coal-fired steam power plant blocks were used. Another aspect of using ANNs in connection with steam power plants is the prediction of $N O_{x}$ emission into the atmosphere [35]. The authors modeled the relationship between the parameters of a $660 \mathrm{MW}$ boiler and the level of $N O_{x}$ emission, while minimizing the level of emissions. ANNs are also used in other types of power plants, e.g. nuclear ones $[28,29]$. However, the idea of using these methods remains similar.

\subsection{Motivations}

The following considerations motivated the approach proposed in this work:

- Diagnostics of the regenerative exchanger is an important task in a steam power plant. Within this diagnostics, mass and energy balances are obtained. This is precise but time-consuming. The reference values obtained using this approach are then compared with those from actual measurements. This is also time-consuming. All this means that the traditional approach to monitoring the regenerative exchanger cannot be used online. The approach proposed in this work is free of this disadvantage. It is based on an online comparison of real values coming from the measurements with the reference values generated in the proposed approach.

- The literature proposes solutions using the idea of comparing values from measurements with reference values. However, these are solutions dedicated to operation in steady power states of a steam power plant. It is important to ensure correct operation of the system in various (including transient) states of a power plant. It seems that the approach proposed in this study meets the following condition: the parameters of the correct operation of heat exchangers can be determined under varying operating conditions. As a consequence, this approach can be applied to a wide range of power load. This is a big advantage because the solutions proposed in the literature can be used for small load changes.
- The proposed approach may be developed in the future. First of all, it can be extended e.g. by using a hierarchical system. Secondly, it may be enriched with additional methods and mechanisms to obtain knowledge describing the dynamics of changes taking place in the regenerative exchanger of a steam power plant. Such knowledge will perfectly complement traditional diagnostic methods.

\subsection{Novelty elements}

The novelty elements of the proposed approach can be concluded as follows:

- An original approach to online monitoring of the regeneration exchanger of a condensation power plant is proposed. Its idea is to monitor a number of selected parameters of an exchanger and to generate reference values for them. Their comparison with the actual values (from measurements) may be the basis for the mechanism of signaling irregularities, i.e. a damage or other unfavorable situations, which need to be prevented in good time. However, in this work we do not focus on this particular issue. The problem of online monitoring of the regeneration exchanger of a condensation power plant has not been considered in the literature so far.

- In the proposed approach the Recurrent Neural Network (RNN, [21, 22, 23, 24, 26, 34]) plays an important role as its purpose is to determine online reference values for a correct operation of the regenerative exchanger. This network can promptly react to rapid changes in the parameters of the monitored system, which undoubtedly is a great advantage. A typical Almeida-Pineda Recurrent Back-propagation algorithm (APRBp, $[1,22,23]$ ) is used to learn RNNs. So far, the literature has not considered the use of dynamic methods (e.g. RNNs) for monitoring the steam plant regeneration exchanger.

- The proposed approach has been tested for different power loads in the range from $120 \mathrm{MW}$ to $190 \mathrm{MW}$ taking into account the actual measurement data during the operation of a $200 \mathrm{MW}$ condensation power plant. Different operating modes related to the range and dynamics of load changes were considered. Several modes, not 
just one, for the whole power load range, were taken into account in order to better compare the results obtained.

\subsection{Structure of the paper}

Section 2 describes the approach to monitoring the regenerative exchanger of a steam plant and its components proposed in this paper. Section 3 presents the results of the simulations. Section 4 summarizes the conclusions.

\section{Description of the proposed ap- proach and its components}

This section introduces issues related to the operation of a steam power plant (Section 2.1.1), and in particular the regeneration exchanger (Section 2.1.2). In addition, an RNN used for its monitoring (Section 2.2.1) and its learning algorithm (Section 2.2.2) are described. Based on these descriptions, the details of the approach proposed in this paper are presented (Section 2.3).

\subsection{Description of the steam power plant}

This article considers a steam power plant with a power of $\mathrm{P}=200 \mathrm{MW}$. Its general diagram is shown in Figure 1 and a simplified diagram in Figure 2 [11]. Section 2.1.1 describes the general working principle of power plant operation, and Section 2.1.2 describes the role of regeneration exchangers in the power plant.

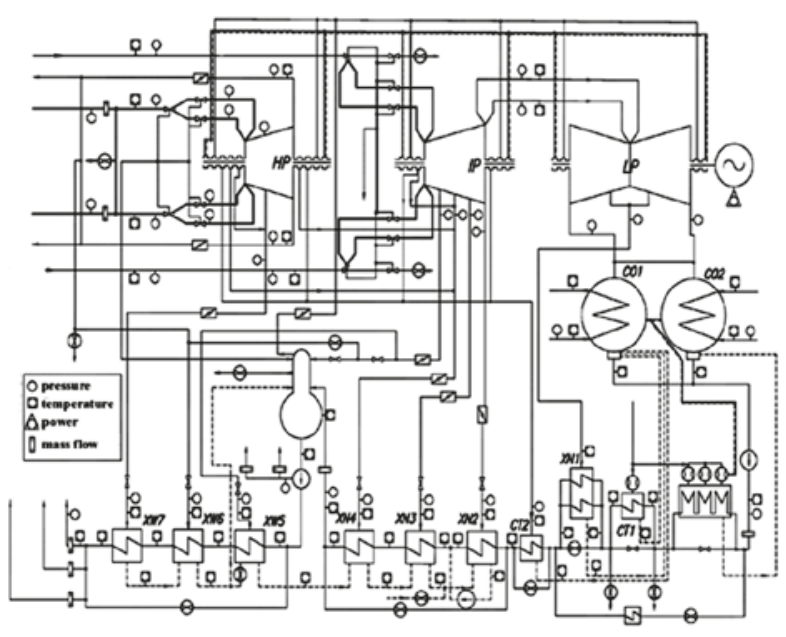

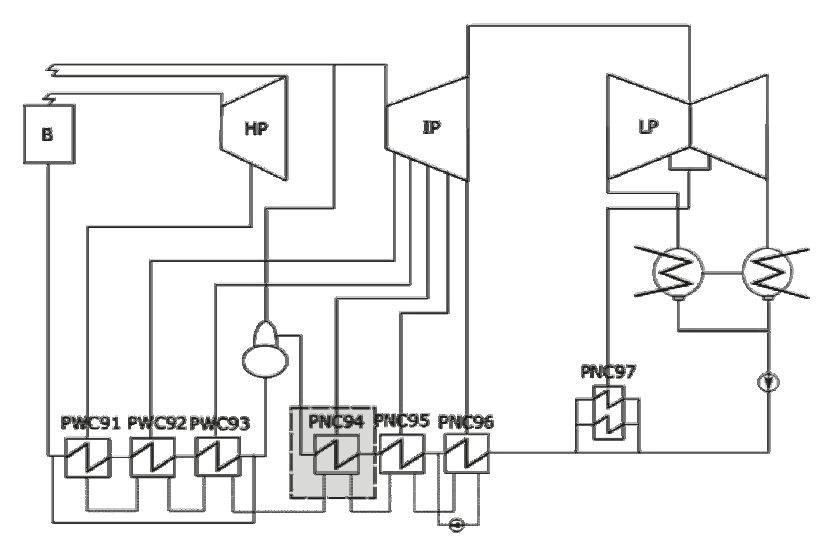

Figure 2. Simplified diagram of a 200MW steam power plant block, whose general detailed diagram is shown in Figure 1. The monitored regeneration exchanger has a grey background.

\subsubsection{Working principle of the steam power plant}

The idea of the block under consideration is as follows. The boiler (B) heats up and evaporates the feed water. It is also supposed to superheat the generated steam. The steam generated in boiler B (so called fresh steam) goes to the part of the high-pressure steam turbine (HP), where it expands. This steam drives the blades of the turbine, which contributes to the production of electricity of a determined power. The steam then goes to the interstage superheater and is superheated there to the fresh steam temperature at the boiler outlet (B). In subsequent stages, the heated steam goes to the hull of the intermediate pressure turbine (IP) and then to the hull of the low pressure turbine (LP). In the IP and LP, the steam expands again and produces energy. After leaving the low-pressure part, the steam is directed to water-cooled condensers in an open system. The condensate then flows successively through the surface regeneration exchangers PNC97-PNC94 supplied by the outlets of the LP low-pressure turbine and IP intermediate-pressure turbine. In each of these exchangers the water is heated up. The water (as a heated working medium) goes to the direct contact heater, where it is deaeratored. Before the water flows back into the boiler (B), it is additionally heated by three surface regeneration exchangers. (PWC93-PWC91).

Figure 1. General block diagram including location of the measuring points. 


\subsubsection{The role of regenerative heat exchangers in a steam power plant}

Regenerative heat exchangers are used in condensing steam blocks to heat up the water feeding the steam boiler. It is a solution commonly used in low and high power systems, and the number of these exchangers is selected individually [16]. The dependence of power on the exchangers operation specifically results from the internal flow of the medium. It depends on the amount of condensate extracted from the blade system for regenerative heat exchangers.

A parameter that describes the operation of the regeneration exchanger well is the final temperature difference $t_{f}$. It is the difference between the saturation temperature of the heating steam extracted from the turbine hull $t_{s}\left(p_{c}\right)$ and the temperature of the heated condensate at the output from the regeneration exchanger $t_{w 2}$ (Figure 3 ).

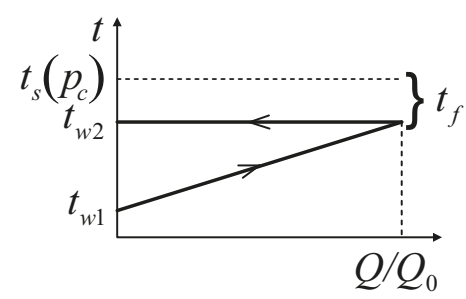

Figure 3. Diagram of temperature changes in the Cascade Closed Feedwater Heater [17].

From the thermodynamic point of view, the final temperature difference $t f$ can be treated as a parameter determining the efficiency of the heat exchange taking place in the regeneration exchanger between the temperature of extracted steam $t_{s}\left(p_{c}\right)$ and the temperature of boiler feed water $t_{w 2}$ :

$$
t_{f}=t_{s}\left(p_{c}\right)-t_{w 2}
$$

In this system, the saturation temperature $t_{s}$ of the extracted steam is determined indirectly by the measured pressure $p_{c}$, while the temperature of the condensate $t_{w 2}$ at the outlet from the exchanger is measured directly.

The simulation part considers in particular the steam powered exchanger from the low-pressure part turbines (LP). In Figure 2, the exchanger is marked with a grey background and has a surface structure, which means that the heat exchange between the heating steam and the condensate occurs through the surface. Therefore, the separated mediums do not mix with each other. In this case the energy balance equation is expressed as follows (Figure 4)

$$
\dot{m} \cdot h_{1}+\dot{m_{w}} \cdot h_{w 1}=\dot{m} \cdot h^{\prime}+\dot{m_{w}} \cdot h_{w 2},
$$

where $\dot{m} \cdot h_{1}$ is the steam heat flow being introduced into the exchanger for heating up the feed water flow of the boiler $\dot{m}_{w}$ with enthalpy $h_{w 1} ; \dot{m}$ is the condensate streams with enthalpy $h^{\prime}$ (generated by cooling the heating steam), and $\dot{m}_{w} \cdot h_{w 2}$ is the water heat flow from the exchanger. Symbols $\left\{\dot{m}, \dot{m_{w}}\right\}$ are mass streams, while $\left\{h_{1}, h_{w 1}, h^{\prime}, h_{w 2}\right\}$ are the enthalpy (heat content).

Although equation (2) is simplified (it does not take into account, e.g. the potential and kinetic energy of the streams), its use for continuous online monitoring of the regeneration exchanger is rather problematic. The aim of the approach proposed in this work is therefore to eliminate the need to use equation (2).

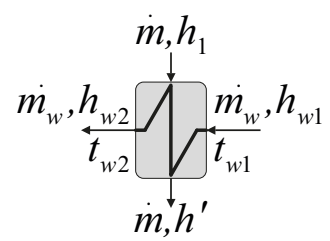

Figure 4. Diagram of a regeneration exchanger with cascade condensate flow [17].

\subsection{Description of the Recurrent Neural Network and its learning algorithm}

There are many recurrent types of neural networks [36]. Their characteristic feature is having their own internal state (memory). It can be implemented in different ways, e.g. by untypical return connections between their layers or by introducing time delays into the network structure. The network state (memory) is used to process input signals and provide dynamic behavior over time. Therefore, RNNs are frequently used to process sequential data, such as text, audio, video and sensor data. For this type of data, the use of a convolutional neural network (CNN, [25]) or a deep neural network (DNN, [12,31]) is not justified because these types of networks are not intended to process 
time-dependent input data. In this work, RNNs with a discrete operating time will be considered [10] due to the specificity of the measurement data. The feedback structure of this network has been selected using the trial and error method.

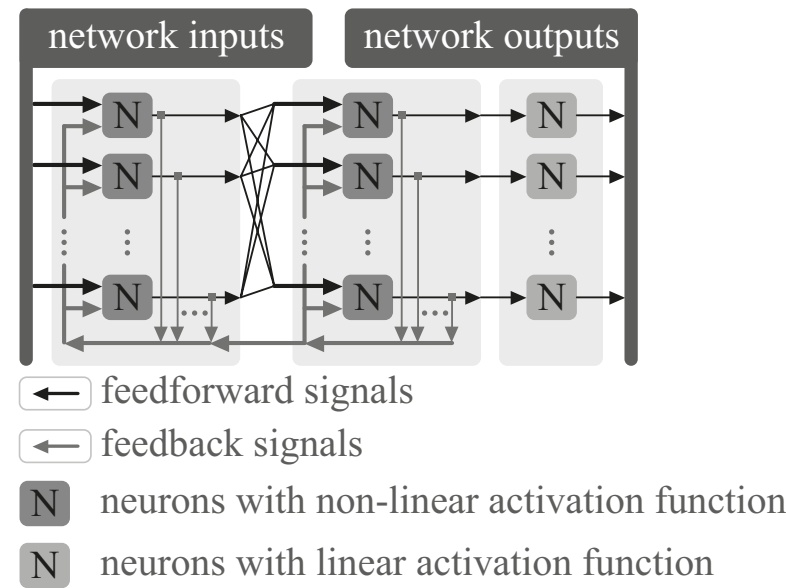

Figure 5. General structure of the Recurrent Neural Network considered in this paper.

\subsubsection{The Recurrent Neural Network structure}

In this work the RNN network is considered, the structure of which is shown in Figure 5. The basic type of network was used to show that the validity of the proposed idea does not depend on the type of the RNN. The comments on this network are as follows:

- It has a three-layer structure. Input signals are delivered to the input layer of the network and do not have to be synchronized with each other. The first two layers contain non-linear neurons, and the output layer contains only linear neurons. It allows us to generate signals outside the range typical for the non-linear activation function used. The structure of a single neuron is typical.

- A single RNN neuron receives signals at its inputs depending to which network layer it belongs (see Figure 5). The first-layer neurons receive network inputs signals (all or selected ones) and feedback signals from the other firstor second-layer neurons (output signals). The second layer neurons receive the output signals from the first layer neurons. In contrast, the third-layer (output) neurons receive only the output signals from the second layer neurons.
- In the neural network under consideration, weights (real) are used to set the strength of connections between neurons. These weights have an additional function in the case of feedback signals, i.e. if the weight value is 0 , the connection associated with this weight is not included in the network.

- An important feature of the considered RNN is the way to connect the feedback signals with neurons. In the simulations it was assumed that this connection can be done in any way except self-connecting. Therefore, some feedback signals may be ignored.

- The activation function in linear neurons is linear with equation $y(x)=x$, while in other neurons it is a hyperbolic tangent of the form

$$
\tanh (x)=\frac{e^{x}-e^{-x}}{e^{x}+e^{-x}}=\frac{e^{2 \cdot x}-1}{e^{2 \cdot x}+1} .
$$

- In order to simplify the visualization of specific connections between RNN neurons, they can be presented as two asymmetrical oriented graphs. The first can illustrate the forward flow of signals (from the inputs to the network outputs). The second graph can illustrate the flow of feedback signals (from the inputs to the outputs of the network). If, in addition, a learning method bases on the back-propagation, then a third graph can also be drawn regardless of the forward and backward graphs. Its task is to illustrate the propagation of error signals from the network outputs to its inputs. These errors are used to correct the values of network weights.

\subsubsection{The Recurrent Neural Network learning algorithm}

The simplest possible method was also used to learn the Recurrent Neural Network (RNN) to demonstrate that the effectiveness of the approach proposed in this work does not depend on the learning algorithm used. Such method is the Pineda and Almeida algorithm [22, 23]. It is a typical algorithm aimed at minimizing the target function according to the gradient descent method. Therefore, the details of this algorithm will not be considered. However, a dependency describing how to modify the network weights is of special importance. Assuming, for the sake of simplicity, that the indexing 
of neurons is continuous and their number is in the range $\langle 1$, Nneurons $\rangle$, the formula for updating the weights takes the following form

$$
w_{r, s}(k+1)=\left(\begin{array}{c}
w_{r, s}(k)+ \\
-\eta \cdot y_{s}(k) \cdot \hat{y}_{r}(k)
\end{array}\right)
$$

where $w_{r, s}$ is the weight for $r$-th input of the neuron $s, y_{s}(k)$ is the output signal from neuron $s$, and $\hat{y}_{r}(k)$ is the output signal from the neuron $r$ connected to the neuron $s$ through the $w_{r, s}$ weight. It is also worth adding that such a way of error propagation in a network is analogous to the method described in [5].

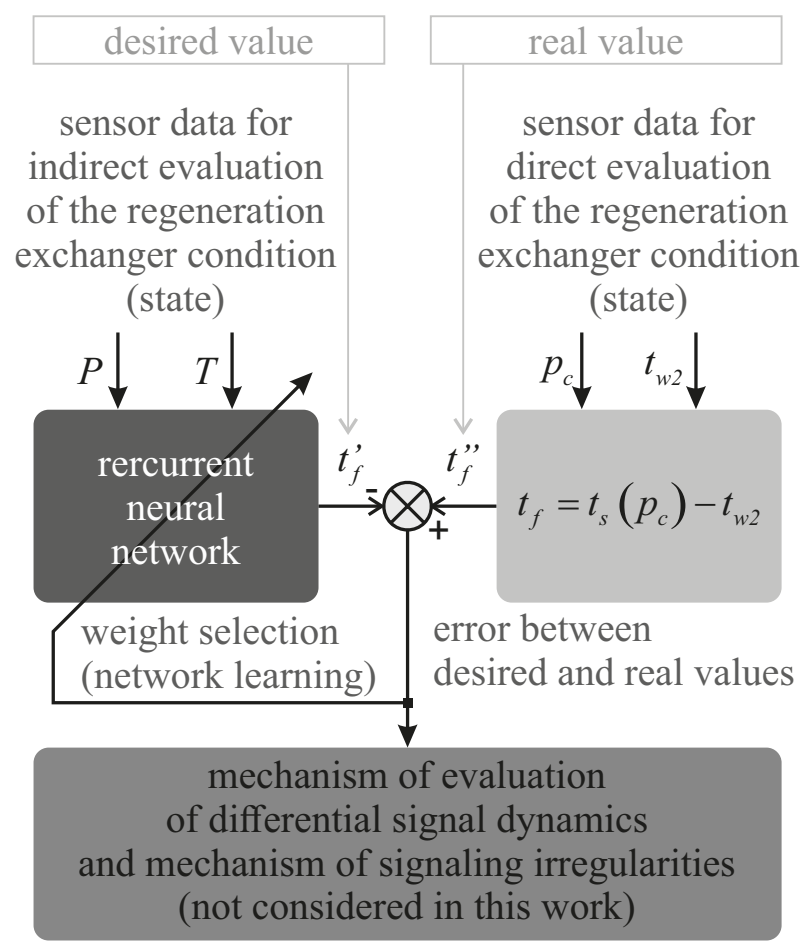

Figure 6. The idea of the proposed approach to online monitoring of the regenerative exchanger in a steam power plant.

\subsection{Description of the proposed approach}

The comments on the proposed approach are as follows:

- The idea of the proposed approach is the practical possibility to determine the final temperature difference $t_{f}$ in two ways (Figure 6). The first way concerns the determination of $t_{f}$ on the basis of the data coming from the sensors not directly related to the operation of the regener- ative exchanger. These are the sensors monitoring the instantaneous value of the power $P$ with which the steam power plant operates and the current temperature $T$ of the condenser cooling water. These two attributes have been selected by the authors from several hundred attributes available in the process of the monitoring of the power plant (see Figure 1). The second way concerns the parameter $t_{f}$ determined on the basis of the data coming from the sensors directly related to the operation of the regeneration exchanger.These are the sensors monitoring the temperature of the extraction steam $t_{s}\left(p_{c}\right)$ and the boiler feed water temperature $t_{w 2}$ (see (1) and Figure 3). In practice, it is of key importance to monitor the differences between the $t_{f}$ determined using both approaches and to react appropriately to these differences in advance. This is shown in Figure 6.

- In the proposed approach, the use of RNNs is crucial for two reasons. Firstly, the ability to generalize the knowledge accumulated in the RNNs and the ability of RNNs to filter the input signals make it possible to ignore fluctuations in the values of the monitored quantities which are typical for power plant operation. Secondly, the ability offered by RNNs to adapt to changing operation conditions allows the RNN used to constantly adapt to different states of the operation of the power plant.

- In the proposed approach, preparing the RNN for work does not require training. This is a key feature that distinguishes the RNN from feedforward networks. After it starts running in the system as shown in Figure 6, the RNN needs actually only a few steps to adjust to the current situation resulting from the values of the sensors $\{P, T\}$ and $\left\{p_{c}, t_{w 2}\right\}$. This consequently results in minimizing the error signal (Figure 6). Then, the monitoring system goes to its standard operation mode. At this stage, the differences between the operation of the regenerative exchanger and the operation expected from the rest of the power plant system can be signalled in advance, which may provide an impulse for an operator intervention.

- The use of the RNN for monitoring the regeneration exchanger creates a possibility of signaling 
undesired operating conditions and taking action to prevent failure (this issue is not considered in this paper).

Table 1. Summary of the considered simulation scenario.

\begin{tabular}{|c|c|c|c|c|c|}
\hline $\begin{array}{c}\text { item } \\
\text { no. }\end{array}$ & $\begin{array}{c}\text { total number } \\
\text { of samples }\end{array}$ & $P_{\min }$ & $P_{\max }$ & $\begin{array}{c}\Delta P \\
\mathrm{MW}\end{array}$ & $\begin{array}{c}\text { main } \\
\text { feature }\end{array}$ \\
\hline I & 2893 & 189.09 & 191.53 & 2.44 & $\begin{array}{c}\text { fixed } \\
\text { load }\end{array}$ \\
\hline II & 2893 & 119.02 & 153.32 & 34.30 & $\begin{array}{c}\text { small } \\
\text { load changes }\end{array}$ \\
\hline III & 401 & 146.97 & 180.05 & 33.08 & $\begin{array}{c}\text { rapid } \\
\text { load changes }\end{array}$ \\
\hline IV & 6186 & 119.02 & 191.53 & $72.39 \begin{array}{c}\text { big } \\
\text { load changes }\end{array}$ \\
\hline
\end{tabular}

Table 2. Summary of the sample values of the signals processed by the RNN according to the concept shown in Figure 6.

\begin{tabular}{|c|c|c|c|}
\hline $\begin{array}{c}\text { item } \\
\text { no. }\end{array}$ & $x_{1}: P \mathrm{MW}$ & $x_{2}: T^{\circ} C$ & $d: t_{f}{ }^{\circ} C$ \\
\hline 1 & 190.3076 & 20.4346 & -6.3784 \\
2 & 190.0635 & 20.2148 & -6.3784 \\
3 & 190.1856 & 20.3613 & -6.1226 \\
4 & 190.1856 & 20.5078 & -6.1970 \\
5 & 190.4297 & 20.5078 & -6.3435 \\
6 & 189.9414 & 20.5811 & -6.5249 \\
7 & 190.6738 & 20.4346 & -6.5621 \\
8 & 190.3076 & 20.4346 & -6.3435 \\
9 & 190.0635 & 20.5811 & -6.3435 \\
10 & 190.0635 & 20.5078 & -6.4507 \\
\hline
\end{tabular}

However, there is also a drawback to the proposed approach. It becomes apparent when there is a noticeable error between the expected and actual values $t_{f}$ (Figure 6). In such a situation an impulse of the differential signal is sent to the monitoring system, but it disappears after a certain time. It disappears because the RNN is being learned to the current situation in the power plant. Therefore, it is necessary to construct a system for evaluating the dynamics of the error signal changing and a system for signaling failures of the regeneration exchanger to make this situation to be detected and accounted for. In the construction of such systems support- ing measuring signals might be taken into account. However, this is an engineering problem and it is not considered in this work.

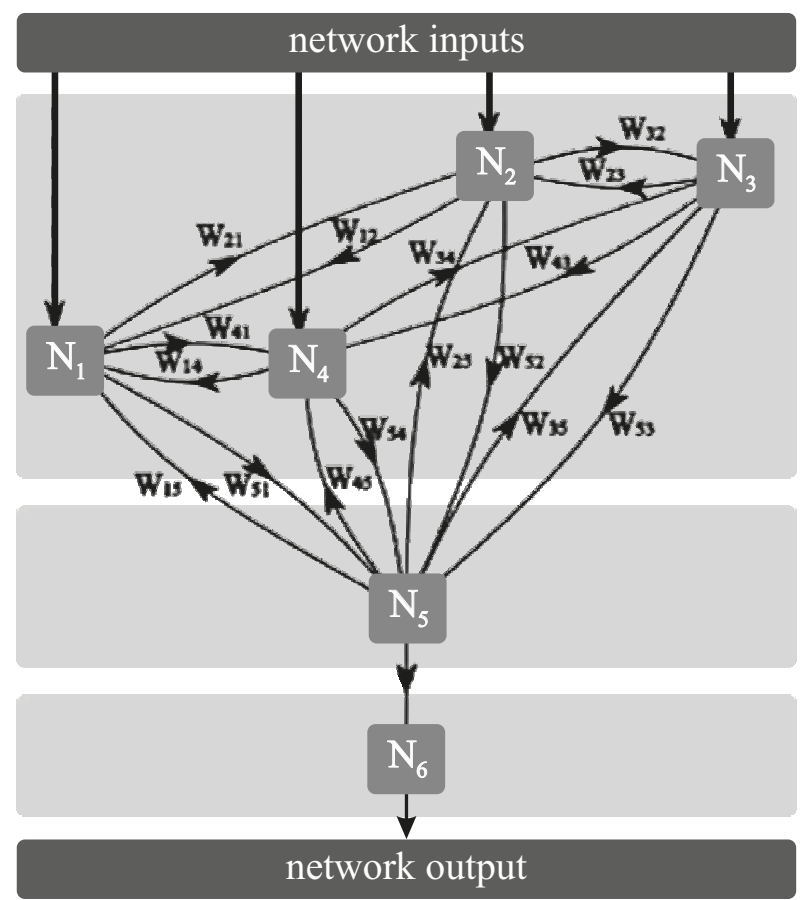

Figure 7. Outline drawings showing the actual and reference values $t_{f}$ for the simulation scenarios under consideration: a) I, b) II, c) III, and d) IV.

Table 3. Comparison of the MSE error values obtained using the methods described in the literature and the one proposed in this paper for considered simulation scenarios I-IV. The best results for each scenario are in bold.

\begin{tabular}{|c|c|c|c|c|}
\hline method & $\begin{array}{c}\text { scenario } \\
\text { I }\end{array}$ & $\begin{array}{c}\text { scenario } \\
\text { II }\end{array}$ & $\begin{array}{c}\text { scenario } \\
\text { III }\end{array}$ & $\begin{array}{c}\text { scenario } \\
\text { IV }\end{array}$ \\
\hline FBNN [4] & 0.0384 & 0.0852 & 0.0887 & 0.1354 \\
RBF [4] & 0.0346 & 0.1125 & 0.1024 & 0.0591 \\
FBNN [11] & 0.0368 & 0.0847 & 0.0884 & 0.1297 \\
FBNN [20] & 0.0368 & 0.0845 & 0.0938 & 0.1268 \\
\hline our result & $\mathbf{0 . 0 0 7 7}$ & $\mathbf{0 . 0 1 4 0}$ & $\mathbf{0 . 0 3 6 8}$ & $\mathbf{0 . 0 0 9 5}$ \\
\hline
\end{tabular}

\section{Simulations}

This Section describes the assumptions made in the simulations (Section 3.1), a comparison of the results obtained using different methods (Section 3.2) and the conclusions of the simulation (Section 3.3). 

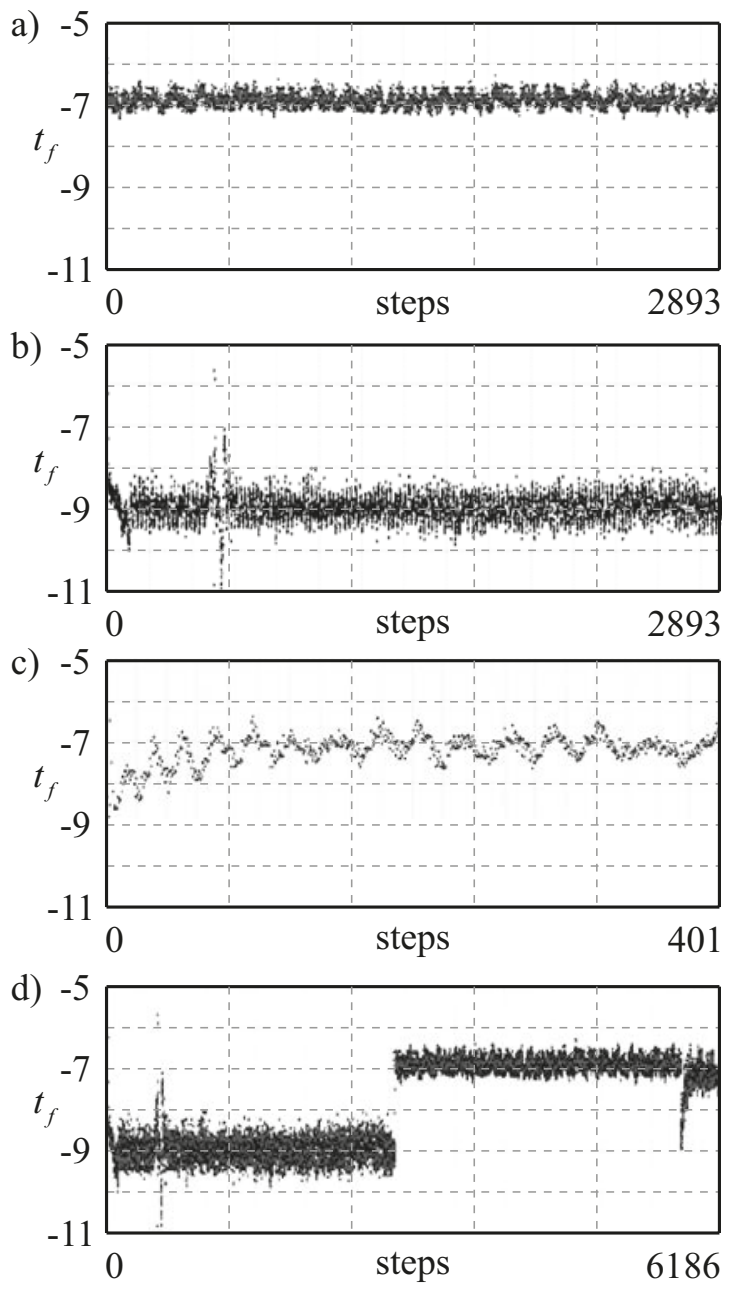

Figure 8. Structure of the Recurrent Neural Network used in the simulation.

\subsection{Simulation assumptions}

The following assumptions were made in the simulations:

- The simulations were carried out with the use of an authorial test environment prepared in $C \#$.

- Four scenarios were considered, which correspond to the typical operating conditions of a condensing power plant, i.e. steady state operation (Scenario I), operation with moderate power changes (Scenario II), operation with abrupt load power changes (Scenario III) and operation with a wide range of power changes (Scenario IV). The details of these options are presented in Table 1.

- The RNN worked in the system considered in 2.3 and shown in Figure 6. Example values of the signals processed by the RNN are shown in Table 2. These signals were not pre-processed (e.g. filtered or reduced).

- The RNN with the structure shown in Figure 3 is used. It is a special form of the network shown in Figure 5. For simplicity, a linear indexing of neurons was adopted. The network structure was selected by the trial and error method. It can be easily seen that some return connections (e.g. between $\mathrm{N}_{3}$ and $\mathrm{N}_{1}$ ) do not occur in the network. All the 0 -weighted connections are not shown in Figure 8 . The weight matrix mapping the structure of the connections between the neurons of the considered network takes the following form

$$
\begin{aligned}
& \mathbf{W}= \\
& {\left[\begin{array}{cccccc}
0 & w_{1,2} & 0 & w_{1,4} & w_{1,5} & 0 \\
w_{2,1} & 0 & w_{2,3} & 0 & w_{2,5} & 0 \\
0 & w_{3,2} & 0 & w_{3,4} & w_{3,5} & 0 \\
w_{4,1} & 0 & w_{4,3} & 0 & w_{4,5} & 0 \\
w_{5,1} & w_{5,2} & w_{5,3} & w_{5,4} & 0 & 0 \\
0 & 0 & 0 & 0 & w_{6,5} & 0
\end{array}\right] .}
\end{aligned}
$$

- The RNN weight matrix (5) was initiated in a random way before the learning process.

- In the simulations, the value of the learning factor $\eta=0.25$ was assumed. To compare the results, a standard mean square error (MSE, [19]) was used.

\subsection{Comparison of the results}

A comparison of the results obtained using the methods considered in the literature can be summarized as follows:

- In order to compare the results obtained using the approach to monitoring the regenerative exchanger of a steam power plant proposed in this work with the results of other authors, the popular methods implemented and tested for intelligent monitoring involved an RBF type network (Radial Basis Function, [4]) and an FBNN type network (Feedforward Back-propagation Neural Network, [11, 20]). By testing the compared methods using the same test procedure, the achieved accuracy of the used methods (see Table 3) can be considered reliable. 
- All the neural network types under consideration (RBF, FBNN and RNNN) performed well with scenario I, in which the load power changes were small. The main problem was, as expected, scenario IV. It corresponded to a situation in which the system had to well reproduce the operation of the regenerative exchanger not only in two different steady states, but also in an unsteady state. In this situation, the feedforward networks were not able to handle the precise generation of $t_{f}$ values. Only the RNN achieved a satisfactory accuracy. This is important because scenario IV is the most relevant from the practical point of view as it occurs in practice most often.

\subsection{Simulation conclusions}

The conclusions of the simulation can be summarized as follows:

- The considered neural network (RNN) mapped with a great accuracy the reality of the regenerative exchanger operating in a condensing block (see Table 3 and Figure 2.3). Due to the large set of samples, Figure 2.3 is purely illustrative. The final temperature difference values $t_{f}$ generated by the RNN differ very little from the actual values. This is demonstrated by the small value of the MSE error (Table 3). This applies to all the considered simulation scenarios (Tab. 1).

- During its operation, the RNN used needed only a few steps to learn how to accurately map the $T$ relationship to $P$ and $T$. This applies to each of the considered simulation scenarios (I-IV, see Table 1). It is possible due to the dynamic nature of the neural network-the possibility of accounting for the relationships changing over time. Because of the small number of steps needed to learn the network and the small value of the MSE error after learning (it is close to 0), the idea of including the time-history of this error in the paper was abandoned.

- A simulation scenario that is difficult to simulate was scenario III, in which there were large fluctuations in load power and hence in $t_{f}$ values (see Figure 2.3.c). This was reflected in the MSE value (see Table 3). However, the easiest scenario to simulate was scenario I, which was discussed in Section 3.2.
- The RNN handled very well scenario IV, which corresponded to two steady states of load power $P$ and an unsteady state. The ability to map this scenario is a great advantage compared to the solutions offered by other authors, which are unable to adequately "switch" between steady operating states of the power plant.

\section{Conclusions}

The paper refers to an important and intensively developed current trend of intelligent monitoring of industrial equipment and processes. In this paper we propose an intelligent approach to the monitoring of the regenerative exchanger in a steam power plant, which is the main input of the paper and it is a good starting point for further research in this area.

In particular, the proposed approach is an alternative to complex calculation and time consuming methods using thermal balance equations. This approach monitors the final temperature difference value for the regenerative exchanger, determining its correct functioning. The Recurrent Neural Network used for monitoring takes into account the dynamics of the process changes in the power plant load power and condenser cooling water temperature.

By taking this dynamic into account, the network under consideration is able to adjust the result of its operation to the current situation, e.g. rapid changes in load power, as soon as after a few steps only. This distinguishes it from other alternatives (e.g. feedforward networks) that are not as flexible as RNNs.

The simulations use real data from a steam power plant that were not pre-processed. The use of the proposed approach allows for a practical and early signaling of abnormal operating conditions of the regeneration exchanger and for launching an appropriate control procedure (this has not been considered in the paper). This is important because the correct operation of the regeneration exchanger determines, among others, the efficiency of the entire power plant.

Our future plans include building a hybrid diagnostic system working online during the operation of the steam block (e.g. monitoring all exchangers), using the proposed approach to monitor other in- 
dustrial processes, using methods for obtaining interpreted knowledge of the changes taking place in the regenerative exchanger of a steam power plant (such knowledge may be complementary to traditional diagnostic methods), and the construction of a dedicated system to interpret the deviations between the monitored quantities.

\section{Acknowledgment}

This paper was financed under the program of the Minister of Science and Higher Education under the name 'Regional Initiative of Excellence' in the years 2019-2022 project number 020/RID/2018/19 with the amount of financing of PLN 12000000.

\section{References}

[1] L. B. Almeida, Backpropagation in nonfeedforward networks, Proc. of the NATO ARW on Neural Computers, Springer Verlag, Heidelberg, 74-91, 1993.

[2] J. Bilski, B. Kowalczyk, A. Marchlewska, J.M. Zurada, Local Levenberg-Marquardt Algorithm for Learning Feedforwad Neural Networks, Journal of Artificial Intelligence and Soft Computing Research, 10(4), 2020.

[3] T. Chmielniak, P. Krzyślak, Comparative analysis of energy potential of three ways of configuration of a condenser power plant thermal cycle, Polish Marit. Res., 15, 30-36, 2008.

[4] T. Chmielniak, H. Łukowicz, Condensing power plant cycle-Assessing possibilities of improving its efficiency, Arch. Thermodyn., 31, 105-113, 2010.

[5] K. Cpałka, Design of interpretable fuzzy systems, Springer, 2017.

[6] P. Deshpande, N. Warke, P. Khandare, V. Deshpande, Thermal power plant analysis using artificial neural network, Proc. of the 3rd Nirma Univ. Int. Conf. Eng. NUiCONE 2012, 1-6, 2012.

[7] R. Dixit, J. Kumar, T. Soota, Modeling of a Thermal Power Plant using Neural Network and Regression Modeling of a Thermal Power Plant using Neural Network and Regression Technique, 2018.

[8] P. Duda, M. Jaworski, A. Cader, L. Wang, On Training Deep Neural Networks Using a Streaming Approach Journal of Artificial Intelligence and Soft Computing Research, 10(1), pp. 15-26, 2020.

[9] A. Gardzilewicz, J. Głuch, M. Bogulicz, R. Walkowiak, M. Najwer, J. Kiebdoj, Experience in
Application of Thermal Diagnostics in the Turow Power Station, Proc. of the Int. Joint Power Generation Conference collocated with TurboExpo 2003 (2003 Int. Joint Power Generation Conference, Atlanta, Georgia, USA), 371-378, 2003.

[10] J. C. Gallagher, S. K. Boddhu, S. Vigraham, A reconfigurable continuous time recurrent neural network for evolvable hardware applications, In Proc. of the 2005 IEEE Congress on Evolutionary Computation, Piscataway, NJ: IEEE, 2005.

[11] J. Głuch, Fault detection in measuring systems of power plants, Polish Marit. Res., 15, 45-51, 2008.

[12] Y. Guo, Y. Liu, T. Georgiou, M. S. Lew, A review of semantic segmentation using deep neural networks, Int. Journal of Multimedia Information Retrieval,2, 1-7, 2017.

[13] R. Isermann, Supervision FDD Methods-An Introduction, Control Eng. Pract., 5, 639-652, 1997.

[14] F. B. Ismail Alnaimi, H. H. Al-Kayiem, Artificial intelligent system for steam boiler diagnosis based on superheater monitoring, J. Appl. Sci., 11, 2011.

[15] A. M. Kler, P. V. Zharkov, N. O. Epishkin, Parametric optimization of supercritical power plants using gradient methods, Energy, 189, 2019.

[16] K. Kosowski, Introduction to the Theory of Marine Turbines, Foundation for the Promotion of Maritime Industry, Gdańsk, 2005.

[17] K. Kosowski, Ship Turbine Power Plants, Fundamentals of Thermodynamical Cycles, Foundation for the Promotion of Maritime Industry, Gdańsk, 2005.

[18] Ł. Kowalczyk, W. Elsner, P. Niegodajew, M. Marek, Gradient-free methods applied to optimisation of advanced ultra-supercritical power plant, Appl. Therm. Eng., 96, 200-208, 2016.

[19] J. K Mohanty., A. Adarsh, P. R. Dash, K. Parida, P. K. Pradhan, Integrated Condition Monitoring of Large Captive Power Plants and Aluminum Smelters, Sound\&Vibration., 53, 223-235, 2019.

[20] T. Niksa-Rynkiewicz, A. Witkowska, Analisis of impact of ship model parameters on changes of control quality index in ship dynamic positioning system, Polish Maritime Research, 2019.

[21] S. Osowski, Signal flow graphs and neural networks. Biological Cybernetics, 70, 387-395, 1994.

[22] F. J. Pineda, Generalization of Back-Propagation to Recurrent Neural Network, The American Physical Society, 59(19), 1988.

[23] F. J. Pineda, Recurrent Back-Propagation and the Dynamical Approach to Adaptive Neural Computation, Neural Computation, 1(2), 161-172, 1989. 
[24] M. A. Ranzato, A. Szlam, J. Bruna, M. Mathieu, R. Collobert, S. Chopra, Video (language) modeling: A baseline for generative models of natural videos. arXiv:1412.6604, 2014.

[25] W. Rawat, Z. Wang, Deep convolutional neural Networks for image classification: A comprehensive review, Neural Computation, 29(9), 1-10, 2017.

[26] A. J. Robinson, F. Fallside, The utility driven dynamic error propagation network. Cambridge: University of Cambridge Department of Engineering, 1987.

[27] L. Rutkowski, Computational intelligence: methods and techniques, Springer Science \& Business Media, 2008.

[28] A. Saeed A. Rashid, Development of Core Monitoring System for a Nuclear Power Plant using Artificial Neural Network Technique, Annals of Nuclear Energy, 144(1), 2020.

[29] M. Saghafia, M. B. Ghofranib, Real-time estimation of break sizes during LOCA in nuclear power plants using NARX neural network, Nuclear Engineering and Technology 51(3), 702-708, 2019.

[30] T. K. Sai, K. A. Reddy, Neural Network Applications in a Power Station, Int. J. Soft Comput., 6, 01-16, 2015.

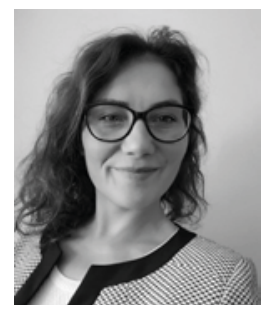

Tacjana Niksa-Rynkiewicz received the M.Sc. and Ph.D. degrees from the Częstochowa University of Technology, Częstochowa, Poland, in 2004 and 2011, respectively. She is currently an Assistant Professor with the Faculty of Ocean Engineering and Ship Technology, Gdansk University of Technology. Dr. Niksa-Rynkiewicz has authored over 10 publications. Her current research interests include computational intelligence, data mining, and expert systems.

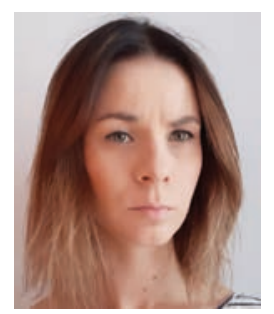

Natalia Szewczuk-Krypa received the M.Sc. degree from the Gdańsk University of Technology, Gdańsk, Poland in 2014. She is currently an Assistant with the Department of Control and Power Engineering. M.Sc. Szewczuk-Krypa has authored over 15 publications. Her current research interests include power plants monitoring and diagnostic, expert systems, and intelligent methods.
[31] P. Sharma, A. Singh, Era of deep neural networks: A review, In Proc. of the 8th Int. Conf. on Computing, Communication and Networking Technologies, Piscataway, NJ: IEEE, 1-5, 2017.

[32] J. Smrekar, D. Pandit, M. Fast, M. Assadi, D. Sudipta, Prediction of power output of a coal-fired power plant by artificial neural network, Neural Comput. Appl., 19, 725-740, 2010.

[33] A. Witkowska., T. Niksa-Rynkiewicz, Dynamically positioned ship steering making use of backstepping method and artificial neural networks, Polish Maritime Research, 2018.

[34] P. J. Werbos, Generalization of backpropagation with application to a recurrent gas market model. Neural Networks, 1(4), 339-356, 1988.

[35] G. Yang Y. Wang, X. Li, Prediction of the NOx emissions from thermal power plant using long-short term memory neural network, Energy, 192(1), 2020.

[36] Y. Yu, X. Si, C. Hu, J. Zhang, A Review of Recurrent Neural Networks: LSTM Cells and Network Architectures, Neural Computation, 31(7), 1235$1270,2019$.

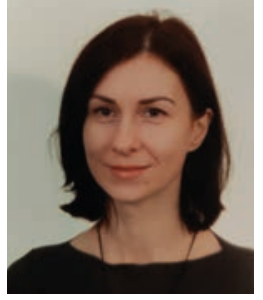

Anna Witkowska holds an M.Sc. in mathematics and computer science from the University of Gdańsk, Poland in 2001. She received her Ph.D. degree from the Technical University of Warsaw in 2011, and the D.Sc. (habilitation) degree in automation, electronic and electrical engineering from the Technical University of Gdańsk, in 2020. Currently she is a professor of the Gdańsk University of Technology, Faculty of Electrical and Control Engineering. Her research interests include automation, especially adaptive and robust control of nonlinear systems as ocean vehicles and control allocation methods in ship dynamic positioning systems.

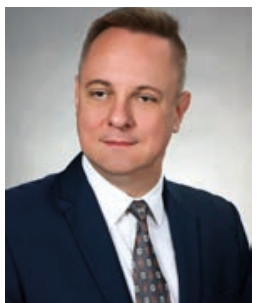

Krzysztof Cpałka was born in Częstochowa, Poland, in 1972. He received the M.Sc. and Ph.D. degrees from the Częstochowa University of Technology, Częstochowa, Poland, in 1997 and 2002, respectively, and the D.Sc. degree in Computer Science from the Systems Research Institute of the Polish Academy of Sciences in Warsaw, Poland, in 2010 
Since 2010, he has been a professor with the Department of Computer Engineering, Częstochowa University of Technology. He is the author or co-author of two books and over 100 refereed papers. He is an Associate Editor in IEEE Transactions on Industrial Informatics and a reviewer for many international scientific journals. Krzysztof Cpałka was a recipient of the IEEE Transactions on Neural Networks Outstanding Paper Award in 2005. His research interests include soft computing, computational intelligence, machine learning, and bio-inspired global optimization algorithms and their applications.

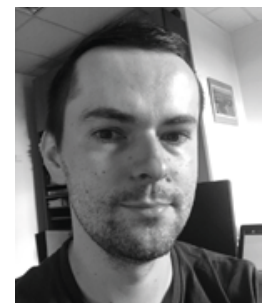

Marcin Zalasiński received the M.Sc. and Ph.D. degrees from the Częstochowa University of Technology, Częstochowa, Poland, in 2009 and 2014, respectively. He is currently an Associate Professor with the Department of Computer Engineering. Dr. Zalasiński has authored over 20 publi- cations. His current research interests include computational intelligence, biometrics, and expert systems.

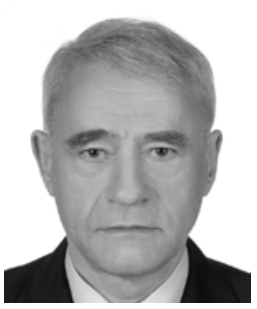

Andrzej Cader is a professor at University of Social Science in Łódź, Poland. He received the Ph.D. degree in biocybernetics and biomedical engineering from the Medical University in Łódź, Poland. His research interests include intelligent systems science based on several architectures such as neural networks and complex systems. $\mathrm{He}$ is also currently engaged in time series analysis and chaos theory. 